\title{
Alterações na Amelogênese e suas Implicações Clínicas: Relatos de casos
}

\author{
Alterations in Amelogenesis and its Clinical Implications/ Cases reports
}

Mirian de Waele Souchois de Marsillac ${ }^{1}$, Alice Mara Rodrigues Batista ${ }^{2}$, Joecí de Oliveira ${ }^{3}$, Maria José de Carvalho Rocha ${ }^{4}$

\begin{abstract}
Alterations in amelogenesis and its clinical implications: Cases reports.

Alterations in amelogenesis can promote disturbances on the development of dental enamel. These alterations during amelogenesis will result in different color and/or structure of dental enamel that may occur isolated or simultaneously. These alterations may involve one tooth or more, yet the causal factors may be local, systemic, genetic or idiopathic. The intensity, extension and the number of teeth presenting alterations in the dental enamel are related to the developmental stage of the enamel dental organ. After the dentist has evidenced a color and/or structural alteration due to developmental defect in amelogenesis it is very important to make a precise diagnosis so that he can proceed with an adequate therapeutic either if it is only a matter of recall appointments, preventive or interceptive approaches. The clinical cases in this study illustrates some of human dental enamel's alterations during amelogenesis and their probable causes based on the patient's clinical history and in the dental literature.
\end{abstract}

Keywords: Dental Enamel; Abnormalities; Dental Fluorosis; Dental Enamel Hypoplasia; Amelogenesis.

\section{Resumo}

Alterações durante a amelogênese podem acarretar distúrbios no desenvolvimento do esmalte dentário. Em consequência a estas alterações o esmalte dentário pode apresentar modificação de cor e/ou que podem ocorrer isoladamente ou de forma simultânea, envolvendo, apenas um ou mais dentes. A intensidade, a extensão e a quantidade de elementos dentários envolvidos pelas alterações no esmalte podem estar relacionadas ao estágio de desenvolvimento do órgão do esmalte dentário. As causas podem ser de origem local, sistêmica, genética ou idiopática. Após a constatação de uma alteração da amelogênese evidenciada por meio da modificação de cor e/ou estrutura do esmalte dentário pelo dentista é importante que haja um diagnóstico preciso para se proceder à terapêutica adequada, seja ela preventiva, interceptativa ou o acompanhamento clínico do caso. Os casos clínicos relatados neste estudo ilustram algumas das alterações da amelogênese no esmalte dentário humano e suas prováveis causas com base na história clínica apresentada pelo paciente e na literatura consultada.

Palavras-chave: Esmalte Dentário; Anormalidades; Fluorose Dentária; Hipoplasia do Esmalte Dentário; Amelogênese.
1 Doutora e Mestre em Odontologia (área de concentração Odontopediatria), Professora Adjunta da Faculdade de Odontologia do Estado do Rio de Janeiro

2 Mestre em Odontologia (área de concentração Odontopediatria) pela Universidade Federal de Santa Catarina

${ }^{3}$ Doutora e Mestre em Odontologia (área de concentração Odontopediatria), Professora Adjunta da Disciplina de Odontopediatria nos Cursos de Graduação e Pós-graduação da Universidade Federal de Santa Catarina

${ }^{4}$ Doutora e Mestre em Odontologia (área de concentração Odontopediatria), ExProfessora Adjunta da Disciplina de Odontopediatria nos Cursos de Graduação e Especialização da Universidade Federal de Santa Catarina

Correspondência: Mirian de Waele Souchois de Marsillac

Endereço: Rua Boulevard 28 de Setembro, 157/ sala 226 - CEP 20551-030, Rio de Janeiro - RJ, Brasil

Fone: (21) 2587-6372

E-mail: mwsm63@yahoo.com.br

Data de Submissão: 20/04/2008

Data de Aceite: 05/02/2010

\section{Introdução}

O esmalte dentário que recobre a coroa anatômica do dente é um tecido de origem ectodérmica. $O$ órgão do esmalte é responsável pela formação do esmalte dentário, também conhecido como amelogênese. Basicamente a amelogênese compreende dois estágios o secretor (fase de secreção) e o de deposição de minerais (fase de maturação). No primeiro estágio ocorre a diferenciação das células do epitélio interno do esmalte em ameloblastos após a deposição de dentina pelos odontoblastos (KATCHBURIAN; ARANA, 1999; MORAN; BROWN; BAWDEN, 2000). A matriz de esmalte depositada nesta fase é parcialmente mineralizada e tem início nas pontas das cúspides e na borda incisal dos dentes, no sentido de dentro para fora. No segundo estágio há uma maior adição de mineral, ao mesmo tempo em que ocorre a remoção de material orgânico e água, resultando em uma fase de maturação. A maturação tem seu início também nas pontas das cúspides e bordos incisais dos dentes, porém ocorre no sentido de fora para dentro (KATCHBURIAN; ARANA, 1999).

Distúrbios locais ou sistêmicos ao órgão do esmalte durante a amelogênese podem produzir alterações no esmalte dentário. Essas alterações do esmalte dentário podem ser relativas a um defeito quantitativo (diminuição da espessura promovendo hipoplasia) ou qualitativo (hipomineralização gerando opacidades) (FÉDÉRATION DENTAIRE INTERNATIONALE, 1992; SUCKLING, 1989; WEERHEIJM; JÄLEVIK; ALALUUSUA, 2001). A fase de atividade do ameloblasto, a duração do distúrbio, e a sua intensidade indicam a inatividade das células de forma temporária ou permanente, determinando a aparência dos três tipos mais comuns de alteração da amelogênese: hipoplasia, opacidade difusa e opacidade demarcada (FÉDÉRATION DENTAIRE INTERNATIONALE, 1992; SUCKLING, 1989).

A causa destes defeitos na formação ou na mineralização do esmalte, durante a amelogênese, pode ser geralmente classificada como: sistêmica, genética, local ou idiopática (BHASKAR, 1978; GUEDES-PINTO, 1997). As influências sistêmicas mais comuns são as deficiências nutricionais, 
endocrinopatias, doenças febris e certas intoxicações químicas. O fator hereditário é provavelmente um distúrbio generalizado dos ameloblastos e os fatores locais afetam dentes isolados, em muitos casos, um único dente (BHASKAR, 1978; BRAIDO, YASSUDA, 1991; GONÇALVES; FERREIRA, 2000).

\section{Revisão da Literatura}

As alterações na amelogênese mais relatadas na literatura odontológica são as hipoplasias, a opacidade demarcada, a opacidade difusa e a amelogênese imperfeita (Al).

\section{Hipoplasia de esmalte}

É uma alteração do desenvolvimento do esmalte que está associada à redução da espessura do mesmo. O esmalte de espessura reduzida pode ter aparência opaca ou translúcida. A hipoplasia pode ocorrer na forma de fosseta ou de sulco, ambos de forma única ou múltipla. Também pode haver a ausência parcial ou completa de esmalte dentário sobre uma área considerável de dentina (FÉDÉRATION DENTAIRE INTERNATIONALE, 1992; SUCKLING, 1989). A hipoplasia é formada durante a fase secretória da amelogênese, após uma agressão de curta duração e a sua severidade determina a extensão da alteração e a translucidez do esmalte parcialmente formado. Tanto fatores locais como sistêmicos podem causar hipoplasias de esmalte. Uma hipoplasia verdadeira deve ser diferenciada de uma perda de esmalte pós-irruptiva associada a um esmalte hipomineralizado (SUCKLING, 1989).

Através de estudos de dureza e do módulo de elasticidade do esmalte hipoplásico Mahoney et al. (2004) constataram que estes valores eram significativamente menores quando comparado a uma região não afetada do mesmo dente, ou com pré-molares sem hipoplasia. Ao microscópio eletrônico de varredura (MEV) os locais com hipoplasia de esmalte apresentam uma estrutura desorganizada com limites pouco demarcados entre os prismas. Esses autores afirmaram que o motivo provável da menor dureza e módulo de elasticidade fosse devido à falta de organização dos cristais de esmalte. Quando a região de transição entre o esmalte sadio e a hipoplasia foi avaliada em um mesmo dente (cerca de 500-600 $\mu \mathrm{m}$ ) as propriedades mecânicas decrescem de forma linear.

\section{Opacidade demarcada}

É uma alteração na translucidez do esmalte onde a porção afetada tem uma superfície lisa, espessura normal e uma delimitação clara com o esmalte normal adjacente. A coloração do esmalte afetado pode ser branca, cor de creme, amarela ou castanha, com a superfície translúcida ou opaca. Essa alteração pode variar em extensão, posição na superfície do dente e distribuição na boca (FÉDÉRATION DENTAIRE INTERNATIONALE, 1992; SUCKLING, 1989). A opacidade demarcada pode apresentar locais de desintegração do esmalte dentário pós-irrupção, onde as margens são irregulares e pontiagudas, diferenciando-as assim de uma hipoplasia (FÉDÉRATION DENTAIRE INTERNATIONALE, 1992; WEERHEIJM, 2004). Uma condição muito citada recentemente é a hipomineralização de molar-incisivo permanente, sendo essa classificada como uma opacidade demarcada (WEERHEIJM; JÄLEVIK; ALALUUSUA, 2001; WEERHEIJM, 2004).

Suckling (1989) verificou que essa alteração foi reproduzida em ovelhas após um trauma aos ameloblastos no estágio precoce de maturação e também ocorreu após uma infecção parasitária na fase de secreção ou no início da fase de maturação do esmalte. Um estudo longitudinal avaliou a predição de um dente permanente apresentar opacidade demarcada em conseqüência de processo carioso no dente decíduo antecessor. Foi constatado que quando o dente decíduo apresentava cárie às chances aumentavam, em mais que o dobro, de seu sucessor permanente apresentar uma opacidade demarcada. Embora o mecanismo pelo qual isto ocorra ainda não esteja completamente elucidado o mesmo está relacionado à presença de infecção periapical do dente decíduo (BROADBENT; THOMSON; WILLIAMS, 2005).

\section{Opacidade difusa}

A opacidade difusa é uma alteração na translucidez do esmalte onde o esmalte defeituoso possui espessura normal. Quando o dente irrupciona o esmalte defeituoso é liso e de cor branca. Esta alteração de translucidez do esmalte pode ocorrer em vários graus e com uma distribuição linear, contínua ou na forma de manchas. Não existe uma delimitação clara da porção afetada como esmalte normal adjacente. Parte ou todo o esmalte poderá ser afetado e a escala de severidade é baseada na extensão e profundidade em que o local afetado. Dependendo da severidade da alteração poderá apresentar pigmentação exógena castanha ou perda de esmalte dentário após sua irrupção (FÉDÉRATION DENTAIRE INTERNATIONALE, 1992; SUCKLING, 1989).

Para evitar a fluorose dentária em crianças de pouca idade a exposição sistêmica de flúor não deve ser maior que 0,07 $\mathrm{mgF} / \mathrm{kg}$ segundo Lima e Cury (2001). Suckling (1989) afirmou que esse defeito do desenvolvimento do esmalte é o resultado de uma agressão contínua (crônica), feita ao longo dos anos, e cuja intensidade é um pouco acima daquela considerada aceitável. Ele também destaca que alguns indivíduos podem apresentar uma alteração no seu metabolismo fazendo com que a presença de uma concentração baixa ou normal de fluoreto, durante a formação do esmalte dental, promova esse tipo de opacidade difusa. Esse autor observou que a opacidade difusa pode ocorrer na fase de maturação ou na de secreção do esmalte e, a severidade dessa alteração aumenta quando o fluoreto é ingerido na fase de secreção. Ainda de acordo com Suckling (1989) outros fatores de ordem sistêmica agindo em um longo período de tempo, durante a amelogênese, como: má nutrição, doenças crônicas ou grandes altitudes, também podem promover opacidades difusas no esmalte dental.

Atualmente o índice de Thylstrup e Fejerskov (1978) é um dos mais utilizados para quantificar o grau de fluorose dentária. Segundo esta classificação os diferentes graus de fluorose dentária podem variar de zero (ausência de fluorose) a nove (perda de quase todo o esmalte dental ficando apenas um halo na cervical $e$ mudança da forma anatomia do dente). No grau TF 4, quando todo o esmalte coronário está afetado pela fluorose este apresenta cor branco calcária e pode haver um início de perda de pequenos pedaços do mesmo. Sendo assim, o dente já seria classificado como grau TF 5 e isto significa que este esmalte não foi somente afetado pelo flúor na época de maturação mas também na de deposição da matriz. A partir do grau TF 4 a severidade das alterações irá aumentar devido ao fato do flúor ter sido administrado na época da deposição de matriz do esmalte.

\section{Amelogênese imperfeita (Al)}

A amelogênese imperfeita é uma má formação do esmalte dental que pode afetar ambas as dentições e é transmitida geneticamente. Essa alteração da amelogênese é hereditária está restrito a estrutura do esmalte sem a presença de outros comprometimentos sistêmicos aparentes. O diagnóstico das amelogêneses geralmente é realizado por meio de estudos clínicos e informação familiar. Existem três tipos de Al (MCDONALD AVERY, 1995): o tipo hipoplásico (caracterizada por falhas na deposição de matriz em forma de sulcos, áreas ou fóssulas); o tipo hipomineralizado (onde o esmalte é macio ao toque e se desprende da dentina como "parede velha"); e o tipo hipomaturado (espessura do esmalte é fina e dura). Já para Witkop (1988) existem 14 tipos de Al sendo, duas formas ligadas ao cromossomo $X$, oito formas autossômicas dominantes e quatro formas autossômicas 
recessivas. Este autor também classificou as diferentes formas de Al segundo o estágio de desenvolvimento dentário em que estas ocorrem. A Tipo I é a hipoplásica; a Tipo II é a hipomaturada; a Tipo III é a hipocalcificada e a Tipo IV é a hipoplásica com taurodontismo.

\section{Objetivo}

O presente estudo relata quatro casos clínicos com o objetivo de elucidar e ilustrar alguns dos tipos mais comuns de alterações na amelogênese observados na clínica odontológica diária.

\section{Relato de Casos Clínicos}

Caso clínico 1 - Paciente com 11 anos de idade, foi indicado para realizar tratamento odontológico na Universidade do Estado do Rio de Janeiro. $\mathrm{Na}$ anamnese, com a mãe, não registrada história de doença ou alteração sistêmica existente naquele momento ou pregressa. Ao exame clínico foram detectadas algumas necessidades de tratamento para lesões de cárie instaladas e também foi observada uma alteração local no elemento 35. Segundo a mãe do paciente o mesmo apresentou várias lesões de cárie, sendo algumas delas extensas, na dentição decídua. O dente que antecedeu o 35 apresentava uma lesão cariosa extensa com fístula. Quando o sucessor permanente irrompeu na boca já apresentava forma alterada, coloração amarelada, ausência de esmalte na face vestibular e espessura delgada de esmalte na face lingual. Também foi constatada uma lesão cariosa na face distal envolvendo a parte da região oclusal. Um possível diagnóstico para esta alteração seria hipoplasia de esmalte, e devido a história pregressa essa hipoplasia também é conhecida por dente de Turner.

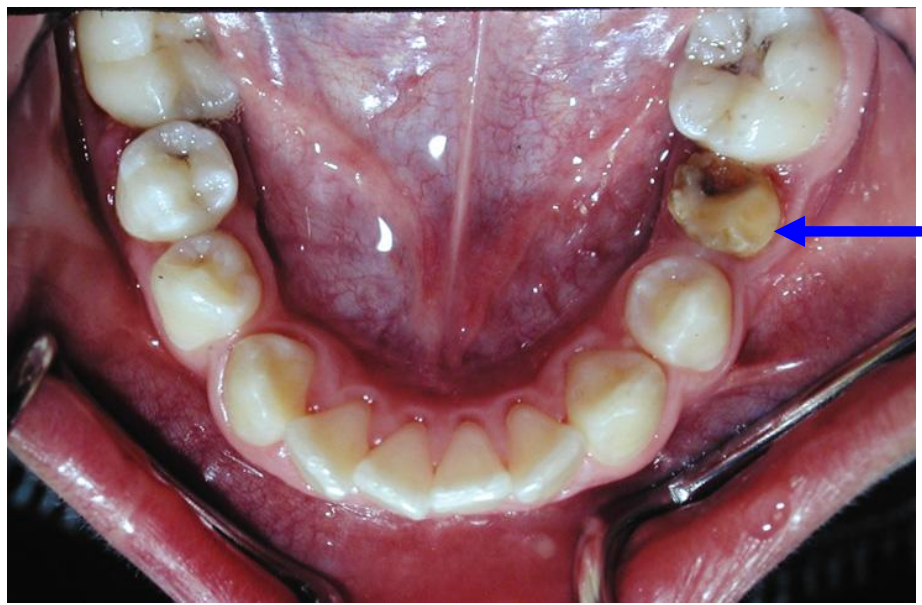

Figura 1: Alteração da amelogênese de ordem local na espessura do esmalte e locais com ausência deste. Essa hipoplasia também e conhecida como dente de Turner. Presença também de lesão cariosa na face distal envolvendo parte da face oclusal.

Caso clínico 2 - Paciente com 08 anos de idade, fo encaminhado por um posto de saúde local para a Faculdade de Odontologia da Universidade do Estado do Rio de Janeiro. Na anamnese o responsável relatou que a criança apresentava lesões extensas de cárie na dentição decídua, porém, não soube precisar os elementos dentários que haviam sido acometidos pela doença após a exodontia. Embora a criança já tivesse recebido tratamento dentário anterior (ex: restauração nos elementos 73 e 85 ) ainda apresentava necessidades preventivas e restauradoras. Ao exame intra-bucal foi verificada uma alteração de cor esbranquiçada, lisa e brilhosa na face vestibular do elemento 34. Como não houve alteração na espessura do esmalte esta seria, provavelmente, uma opacidade demarcada.
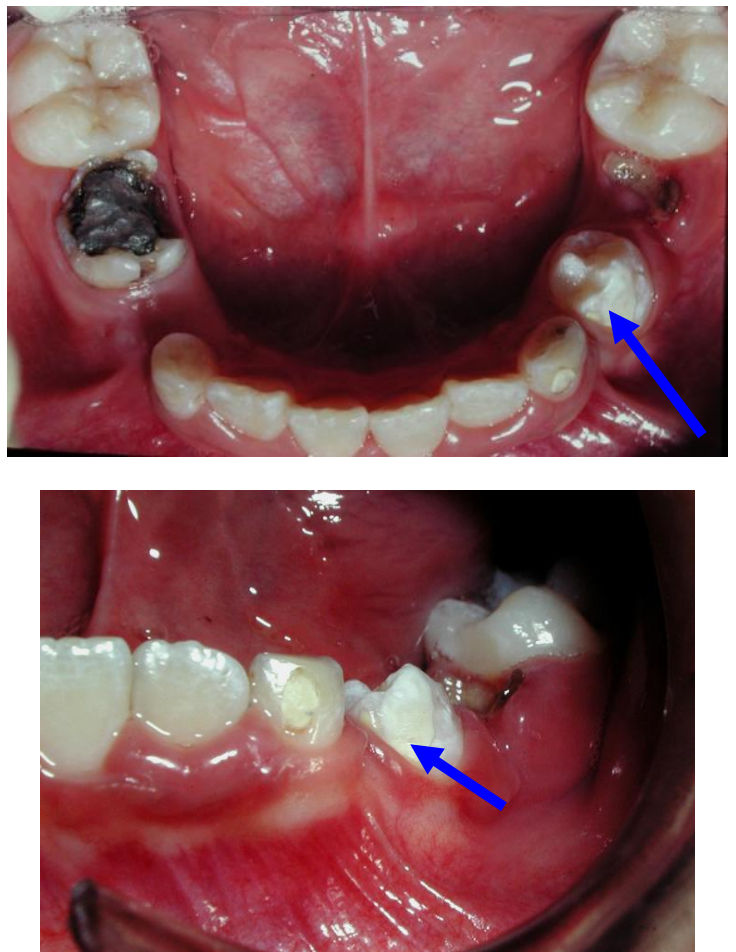

Figura 2: Alteração da amelogênese onde não há evidencia de comprometimento na espessura do esmalte. Há uma modificação de cor, branca cremosa de superfície lisa, localizada na vestibular do elemento dentário 34 sugestiva de opacidade demarcada.

Caso clínico 3 - Paciente com 10 anos de idade, procurou atendimento odontológico na Faculdade de Odontologia da Universidade do Estado do Rio de Janeiro. Ao exame intra-bucal constatou-se uma alteração de coloração do esmalte dental de vários dentes e em ambas arcadas da criança. Linhas brancas e leitosas, porém, com brilho foram observadas em muitas partes das superfícies dentais envolvidas. Em determinados locais, estas linhas se fundiam formando áreas nebulosas, entretanto, nem a criança nem a família haviam percebido esta alteração de cor. Segundo dados fornecidos pela mãe do paciente essa criança fazia uso de complexos vitamínicos contendo fluoretos e utilizava dentifrícios fluoretados desde a irrupção dos primeiros dentes. Somado a isso, essa criança ainda estava exposta à ingestão da água de abastecimento fluoretada da cidade do Rio de Janeiro. Este aspecto clínico sugere um diagnóstico de fluorose.

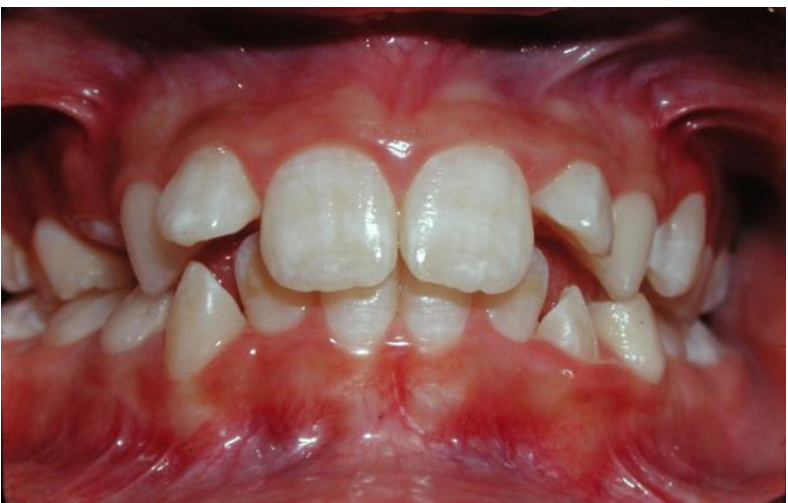

Figura 3: Alteração da amelogênese observada em todas as faces dos dentes permanentes afetados detectada por meio de linhas e áreas esbranquiçadas sem 0 envolvimento da espessura do 
mesmo. Essa alteração é sugestiva de uma opacidade difusa produzida pela ingestão crônica de fluoretos.

Caso clínico 4 - Paciente com 15 anos de idade, procurou atendimento odontológico na Faculdade de Odontologia da Universidade do Estado do Rio de Janeiro porque estava insatisfeita com a sua oclusão e gostaria de realizar um tratamento ortodôntico. Ela nunca tinha se queixado da aparência dos seus dentes e, segundo a mesma, na família da mãe dela também havia pessoas com os dentes semelhantes ao dela. Ao exame clínico verificou-se que a espessura do esmalte dental era delgada e com a textura firme. Devido à diminuição na espessura do esmalte a coloração da dentina tornou-se mais evidente deixando os dentes com um aspecto amarelado. A provável causa da do intenso desgaste do fino esmalte dental nos dentes posteriores foi devido ao desgaste do mesmo em função da mastigação. $O$ aspecto clínico e a história familiar relatada sugeriram um diagnóstico de amelogênese imperfeita.

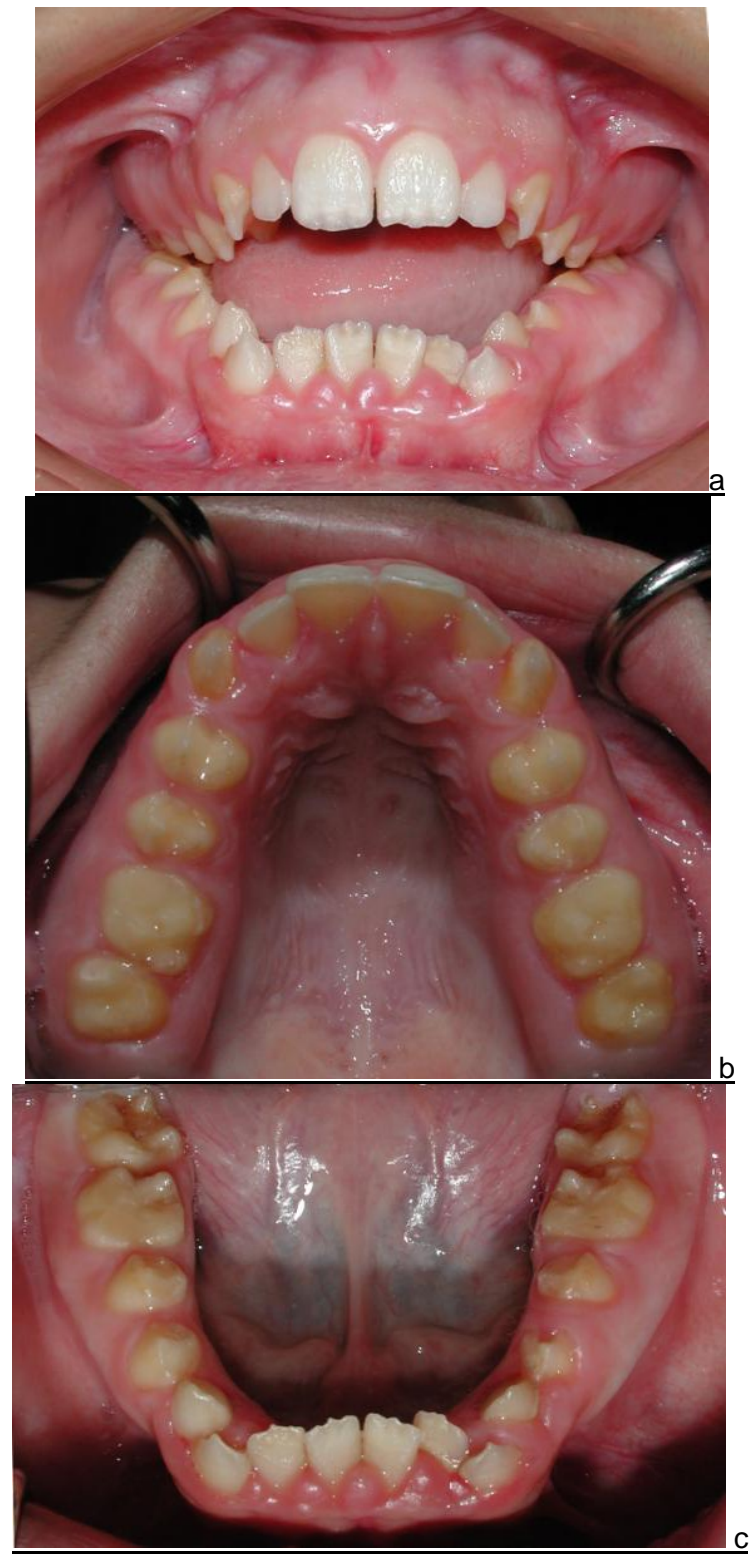

Figura 4a, b e c: Alteração da amelogênese abrangendo todas as faces de toda dentição permanente. A espessura do esmalte dentário apresentava-se diminuída, porém com consistência firme, sugerindo o diagnóstico de amelogênese imperfeita.

\section{Discussão}

No caso clínico 1 há diminuição da espessura de esmalte dental no elemento 35. O diagnóstico para esta alteração foi dado como sendo de hipoplasia local devido à cárie e degeneração pulpar do elemento 75. A má formação do dente 35 ocorreu, mais provavelmente, em um estágio precoce da secreção do esmalte.

A hipoplasia do esmalte é causada por fatores ambientais ou locais e podendo acometer grupos de dentes ou apenas um dente (NEVILLE et al.,1998; PINHEIRO et al., 2003; REGEZI; SCIUBA, 2000; SUCKLING, 1989). A literatura determina diversas causas para esta alteração, originadas por condições ambientais, como: deficiência das vitaminas A, C e D; doenças exantemáticas (sarampo, varicela e escarlatina); hipocalcemia; infecção ou traumatismo local; parto prematuro; distúrbios neonatais; baixo peso ao nascimento ou ainda causas idiopáticas. Contudo, a maioria das hipoplasias seria de origem desconhecida (BRAIDO; YASSUDA, 1991; DIAB; ELBADRAWY, 2000; Hine e Levy, 1987; Li; NAVIA; BIAN, 1995; PINHEIRO et al., 2003; SHAFER; HINE; LEVY, 1991; SUCKLING, 1989).

O esmalte hipoplásico representa um registro das agressões significativas recebidas durante o desenvolvimento do dente bem como a época e duração do estímulo sobre os ameloblastos tem um grande impacto na localização e aspecto desse defeito promovido pela alteração no desenvolvimento esmalte (SUCKLING, 1989). A partir da posição dessas alterações no dente irrompido pode ser possível determinar a idade aproximada da criança no momento da disfunção do órgão do esmalte (ALEXANDRE et al., 2000; DUARTE et al., 2001).

Estudos clínicos mostram a freqüência e a localização das opacidades e das hipoplasias na dentição decídua e na permanente (DAVIES, 1998; LI; NAVIA; BIAN, 1995; MACEDO et al., 2003; SEOW; HUMPHRYS; TUDEHOPE, 1987). Em uma amostra de 1.344 crianças chinesas foi verificado que a maioria dessas alterações da amelogênese ocorria na face vestibular dos dentes sendo que $1,6 \%$ eram opacidades e $22,2 \%$ hipoplasias de esmalte (Li; NAVIA; BIAN, 1995).

Uma pesquisa desenvolvida em Uberlândia (MG - Brasil) constatou que a prevalência de alterações da amelogênese, hipoplasia e hipomineralização, nos dentes decíduos e permanentes de crianças prematuras $(n=110)$ foi de $78,18 \%$ e nas crianças a termo $(n=94)$ de $10,63 \%$ (MACEDO et al., 2003).

Segundo Seow, Humphrys e Tudehope (1987) e Davies (1998) crianças com baixo peso ao nascer ou que tenham apresentado doenças sistêmicas no período neonatal são mais propensas a terem hipoplasia de esmalte também conhecida como hipoplasia linear do esmalte. Li, Navia e Bian (1995) verificaram que crianças prematuras apresentavam quatro vezes mais hipoplasia de esmalte nos dentes decíduos que aquelas nascidas a termo.

Segundo Seow, Humphrys e Tudehope (1987) e Horowitz (1998) as áreas de hipoplasia linear de esmalte serviriam como locais para retenção de biofilme dentário. A colonização precoce da boca da criança com Streptococcus do grupo mutans, o uso prolongado da mamadeira com soluções contendo sacarose e/ou da amamentação materna, principalmente a noturna, são fatores importantes na predisposição da cárie de acometimento precoce ou cárie da primeira infância.

Dentro dos distúrbios locais é importante ressaltar que um problema pulpar (inflamação/infecção) com repercussões perirradiculares ou uma luxação intrusiva de um dente decíduo pode promover desde uma opacidade a uma hipoplasia ou ainda a paralisação ou involução no desenvolvimento do germe do dente permanente sucessor, dependendo do estágio de formação em que o último se apresenta (ALEXANDRE; CAMPOS; OLIVEIRA, 2000; ANDREASEN; ANDREASEN, 2001; BROADBENT; THOMSON; WILLIAMS, 2005; CORDEIRO; ROCHA, 2005). O tipo de traumatismo sofrido no dente decíduo, geralmente determina o tipo e o grau da alteração da amelogênese gerado no dente permanente. A luxação intrusiva de um dente decíduo pode 
promover um risco maior de alterar o desenvolvimento do esmalte do dente permanente sucessor. Isso é devido a grande proximidade da raiz do decido com a coroa dentária do seu sucessor permanente (ALEXANDRE; CAMPOS; OLIVEIRA, 2000; DUARTE et al., 2001; ANDREASEN; ANDREASEN, 2001).

A hipoplasia do esmalte no germe do permanente sucessor só irá ocorrer enquanto o esmalte estiver sendo secretado. A esta entidade denomina-se de dente de Turner (SHAFER; HINE; LEVY, 1991). Após a formação completa da coroa dentária, estágio 6 de Nolla (NOLLA, 1960), não haverá mais risco de hipoplasia podendo haver, no entanto, alteração de cor na coroa que está na fase de maturação do esmalte. A alteração de cor na translucidez do esmalte dentário devido a trauma ou infecção local durante a fase de maturação do esmalte é conhecida como opacidade demarcada. Provavelmente isto é o que ocorreu no caso clínico 2. Quando foi detectada a alteração de coloração do elemento 34 o acompanhante foi esclarecido de que aquela era uma possível seqüela da degeneração pulpar, decorrente de cárie, no dente decíduo sucessor sobre o germe do permanente. Uma doença inflamatória periapical pode provocar uma opacidade demarcada no seu permanente sucessor, caso a sua coroa já esteja totalmente formada, mas ainda em fase de maturação. Como descrito por Broadbent, Thomson e Williams (2005), as chances de um dente permanente apresentar uma opacidade demarcada devido ao processo carioso de um dente decíduo, aumentam em mais de o dobro. Certamente este paciente apresentava diversas lesões cariosas extensas pois apenas na arcada inferior verificouse a perda precoce do elemento 84 , resto radicular do 75 e uma restauração extensa do 85 .

A alteração de cor nos dentes observada no caso clínico 3 foi diagnosticada, clinicamente, como sendo uma opacidade difusa mais conhecida como fluorose. Através dos dados fornecidos pela mãe do paciente esta alteração foi causada pela ingestão de diferentes fontes de fluoretos pela criança desde uma tenra idade (cerca de 8 meses de idade). O grau de fluorose, segundo Thylstrup e Fejerskov (Fejerskov et al., 1994), para este caso seria 3. A extensão e severidade da fluorose variam de acordo com da dose de fluoreto ingerida durante o período de maturação ou de secreção do esmalte dental de cada grupos de dentes. Como não houve comprometimento na formação da matriz do esmalte no caso clínico relatado esta alteração deve ter ocorrido durante a fase de maturação e não de secreção.

São vários os relatos clínicos de fluorose na literatura odontológica. No Brasil, esta opacidade difusa provocada pela ingestão de fluoretos na fase de secreção ou de maturação do esmalte, também foi analisada em termos de severidade e de prevalência. Oliveira e Milbourne (2001) realizaram uma pesquisa com 266 escolares de 7 a 12 anos de idade, em uma escola pública do Rio de Janeiro. A prevalência de fluorose relatada nos dentes avaliados, apenas os incisivos superiores, foi de $7,9 \%$. A intensidade variou de 1 a 3 do índice de Thylstrup e Fejerskov (TF), sendo que $77 \%$ dos dentes afetados tiveram registros grau 1 .

Outra pesquisa sobre fluorose realizada em Marília, São Paulo, foram examinadas 994 crianças de 3 a 6 anos de idade. Os graus de fluorose encontrados foram de $2,8 \%$ muito leve, $9 \%$ leve, $0,3 \%$ moderado e $0,2 \%$ severa. O porcentual de crianças com fluorose aos três anos de idade foi de $4,8 \%$, para os quatro anos de idade $6,7 \%$ e para as crianças de cinco e seis anos de idade foi de $10,3 \%$. Devido a esta baixa prevalência de fluorose em decíduos os autores afirmaram que esta não constituía um problema de saúde pública (ALVES et al., 2002).

É interessante considerar que a percepção das pessoas com fluorose em graus abaixo de TF 4 muitas vezes não altera os padrões aceitos pelas mesmas em termos de estética. Para que a fluorose seja considerada um problema de saúde pública para uma dada população seria importante considerar a gravidade das alterações, em termos de perda de substância e de dentes afetados, a prevalência desta e da diminuição da qualidade de vida das mesmas.
Dentre as alterações da amelogênese a $\mathrm{Al}$ é a única que ocorre por transmissão genética. O diagnóstico clínico do caso clínico 4 foi de amelogênese imperfeita do tipo hipomaturado. Isto porque, segundo McDonald e Avery (1995), o tipo hipomaturado possui um esmalte de espessura fina e firme. Entretanto, a paciente não estava preocupada com esta alteração e sim com a sua má oclusão. Foi esclarecido à paciente que, para realizar um tratamento ortodôntico seriam necessários uma série de procedimentos preventivos e restauradores, exames complementares e uma abordagem multidisciplinar. Os diferentes tipos de Al podem ser diferenciados clinicamente, porém para um diagnóstico definitivo deve ser realizada uma pesquisa genética. Esta alteração acomete todos os dentes de uma dentição e poderá afetar ambas as dentições (MCDONALD; AVERY, 1995).

Nenhum dos quatro pacientes e/ou seus familiares queixavam-se de problemas diretamente relacionados às alterações da amelogênese sobre a sua qualidade de vida. É preciso lembrar que nem todas as alterações da amelogênese necessitam de intervenção profissional (FÉDÉRATION DENTAIRE INTERNATIONALE, 1992). As implicações clínicas dos quatro casos clínicos apresentados envolveram tomadas de decisões individualizadas quanto às necessidades preventivas para cárie dentária, periodontopatias, má oclusão e não apenas para os locais com alterações da amelogênese. Portanto, com base na Odontologia de Mínima Intervenção (midentistry.com) os pacientes e suas famílias foram envolvidos no processo de capacitação para cuidados com a sua boca. Quando houve necessidade de tratamento restaurador optou-se também por técnicas de mínima intervenção, tanto para os dentes cariados como para aqueles que apresentavam alteração na amelogênese com risco de acúmulo de biofilme. Com exceção dos dentes com alterações de opacidade demarcada (caso clínico 2, elemento dentário 34) e da opacidade difusa (caso clínico 3, todos os dentes permanentes) optou-se por um tratamento onde $o$ paciente, sua família e o profissional chegassem a um consenso.

Algumas alterações da amelogênese devem receber acompanhamento profissional com intervalos pequenos devido ao risco da perda de esmalte pós-irruptiva (determinados tipos de hipomineralizações) ou devido ao desgaste do esmalte fino (alguns tipos de hipoplasias e de amelogênese imperfeita). Para minimizar essa perda de esmalte Weerheijm (2004) indica procedimentos preventivos e interceptativos como a correta limpeza dos dentes, aplicação tópica de verniz fluoretado e selantes ionoméricos. Ele considera que o alívio da dor, quando presente, deve ser o principal foco seguido da viabilização da função do dente dentro do esperado em termos do seu ciclo vital.

O tratamento restaurador para hipoplasia e a hipomineralização pode variar conforme a extensão e o grau de comprometimento do esmalte dentário. Seow e Amaratunge (1998) demonstraram que o condicionamento ácido do esmalte dentário pode ser utilizado em restaurações adesivas para os casos de amelogênese imperfeita. Entretanto, o elevado número de falhas dessas restaurações em dentes com $\mathrm{Al}$ quando, comparado a dentes com esmalte normal, podem estar relacionados com outros fatores como as fraturas no esmalte com alteração na amelogênese ou na dentina. Hiraishi, Yiu e King (2008) constataram que a adesão à dentina hipermineralizada de dentes decíduos com Al ficava comprometida quando realizada com condicionamento ácido lavagem e aplicação de sistema adesivo. aumento do tempo de condicionamento ácido nesses dentes também não melhorou a adesão a essa dentina hipermineralizada.

Weerheijm (2004) cita os materiais adesivos como opção de tratamento para áreas com perda de esmalte devido a uma alteração na amelogênese. É importante que essas restaurações tenham suas margens em esmalte sadio, porém em alguns casos isso seja de difícil detecção clínica. Ele também considera que restaurações semi-definitivas (coroas de aço ou restaurações fundidas cimentadas com adesivos) possam ser boas alternativas. Por vezes a última opção da exodontia com correção ortodôntica se faz necessária. 


\section{Conclusão}

Os casos clínicos relatados neste estudo ilustram algumas das alterações da amelogênese no esmalte dentário humano e suas prováveis causas com base na história clínica apresentada pelo paciente e na literatura consultada. Assim, foi possível identificar clinicamente quatro diferentes tipos alterações da amelogênese e suas implicações clínicas: a hipoplasia de esmalte, a amelogênese imperfeita, a opacidade demarcada e a difusa (fluorose dentária).

\section{Referências}

AINAMO, J.; CUTRESS, T.W. An epidemiological index of developmental defects of dental enamel (DDE Index). Int. Dent. J., London, v. 32, no. 2, p. 159-167, June 1982.

ALEXANDRE, G.C.; CAMPOS, V.; OLIVEIRA, B.H. Luxação intrusiva de dentes decíduos. R. Assoc. Paul. Cir. Dent., São Paulo, v. 54, n. 3, p. 215-219, maio/jun. 2000.

ALVES, N.C. et al. Prevalência de fluorose dentária na dentição decídua em crianças participantes de programas de prevenção e programas de promoção de saúde no município de Marília. R. Fac. Odontol. Bauru, v. 10, n. 3, p. 156-163, jul./set. 2002.

ANDREASEN, J.O.; ANDREASEN, F.M. Fundamentos de traumatismo dental: guia de tratamento passo a passo. 2. ed. Porto Alegre: Artmed, 2001. p. 141-154.

BHASKAR, S.N. Histologia e embriologia oral de Orban. 10. ed São Paulo: Artes Médicas, 1978. p. 51-110.

BRAIDO, C.A.; YASSUDA, L.Y.W. Anormalidades de calcificação dentária. Pediatr. Mod., São Paulo, v. 26, n. 2, p. 103-116, mar./abr. 1991.

BROADBENT, J.M.; THOMSON, W.M.; WILLIAMS, S.M. Does caries in primary teeth predict enamel defects in permanent teeth? A longitudinal study. J. Dent. Res., Chicago, v. 84 , no. 3, p. 260 264, Mar. 2005.

CORDEIRO, M.M.R.; ROCHA, M.J.C. The Effects of periradicular inflamation and infectionon on a primary tooth and permanent successor. J. Clin. Pediatr. Dent., Birmingham,v. 29, no. 3, p. 193200, Spring 2005.

DAVIES, G.N. Early childhood caries--a synopsis. Community

Dent. Oral Epidemiol., Copenhagen, v. 26, no. 1, p. 106-116,

1998. Supplement.

de Pró-reitoria e Pós-Graduação da Universidade Federal de Uberlândia, Minas Gerais, v. 2, n. 2, 2003. Disponível em: <http://www.propp.ufu.br/revistaeletronica/index.html> Acesso em: 4 dez. 2007.

MAHONEY, E.K. et al. Mechanical properties across hipomineralized/hipoplastic enamel of first permanent molar teeth. Eur. J. Oral Sci., Copenhagen, v. 112, no. 6, p. 497-502, Dec. 2004

MCDONALD, R.E.; AVERY, D.R. Alterações congênitas e adquiridas dos dentes e estruturas bucais associadas. In: Odontopediatria. 7. ed. Rio de Janeiro:

Guanabara Koogan, 2001. p. 85-96.
DIAB, M.; ELBADRAWY, H.E. Intrusion injuries of primary incisors. Part III: effects on the permanent successors. Quintessence Int. Berlin, v. 31, no. 6, p. 377-384, June 2000.

DUARTE, D.A. et al. Caderno de Odontopediatria: lesões traumáticas em dentes decíduos: tratamento e controle. São Paulo: Santos, 2001. p. 37-42.

FÉDÉRATION DENTAIRE INTERNATIONALE. Commission on Oral Health, Research and Epidemiology. A review of the developmental defects of enamel index (DDE Index). Int. Dent. J. London, v. 42, no. 6, p. 411-426, Dec. 1992.

GONÇALVES, A.F.; FERREIRA, S.L.M. Defeitos hipoplásicos do esmalte dentário. R. Odont. Univ. Santo Amaro, São Paulo, v. 5 , n. 1 , p. $13-20$, out. 2000.

GUEDES-PINTO, A.C. Odontopediatria. 6.ed. São Paulo: Santos, 1997. p.1-15.

HIRAISHI, N.; YIU, C.K.; KING, N.M. Effect of acid etching time on bond strength of an etch-and-rinse adhesive to primary tooth dentine affected by amelogenesis imperfecta. Int. J. Paediatr.

Dent., Oxford, v. 18, no. 3, p. 224-230, May 2008.

HOROWITZ, H.S. Research issues in early childhood caries.

Community Dent. Oral Epidemiol., Copenhagen, v. 26, p. 67-81, 1998. Supplement 1.

KATCHBURIAN, E.; ARANA, V. Histologia e embriologia oral: texto-atlas e correlações clínicas. São Paulo: Panamericana, 1999. p. 237-279.

LI, Y.; NAVIA, M.J.; BIAN, J.Y. Prevalence and distribution of developmental enamel defects in primary dentition of chinese children 3-5 years old. Community Dent. Oral Epidemiol., Copenhagen, v. 23, no. 2, p. 72-79. Apr. 1995.

LIMA, Y.B.O.; CURY, J.A. Ingestão de flúor por crianças pela água e dentifrício. R. Saúde Pública, São Paulo, v. 35, n. 6, p. 576-581, dez. 2001.

MACEDO, L.A. et al. Defeitos de esmalte em decíduos em dentes decíduos e permanentes em crianças prematuras e em crianças entubadas. R. Horizonte Científico: Revista on-line da Faculdade

MIDENTISTRY. Johannesburg: Minimal Intervention Dentistry (MI), 2006. Disponível em: < http://www.midentistry.com/forum.html >. Acesso em: 6 jun. 2009.

MORAN, R.A.; BROWN, E.M.; BAWDEN, J.W. Immunohistochimical localization of Gaq, PLC $\beta$, Gai1-2, PKA, and the endothelin $\mathrm{B}$ and extracelular $\mathrm{Ca}^{2+}$-sensing receptors during early amelogenesis. J. Dent. Res., Chicago, v. 79, no. 11, p. 18961901, Nov. 2000. 
SUCKLING, G.W. Developmental defects of enamel - Historical and present - day perspectives of their pathogenesis. Adv. Dent. Res., Washington, v. 3, no. 2, p. 87-94, Sept. 1989.

THYLSTRUP, A.; FEJERSKOV, O. Clinical appearance of dental fluorosis in permanent teeth in relation to histological changes. Community Dent. Oral Epidemiol., Copenhagen, v. 6, no. 6 , p. 315-328, Nov. 1978.

WEERHEIJM, K.L. Molar hypomineralization (MIH): clinical presentation, aetiology and management. Dent. Update, London, $v$. 31, no. 1, p. 9-12, Jan./Feb. 2004.

WEERHEIJM, K.L.; JÄLEVIK, B.; ALALUUSUA, S. Molar-incisor hypomineralization. Caries Res., New York, v. 35, no. 5, p. 390391, Sept./Oct. 2001.

WITKOP, C.J. Amelogenesis imperfecta, dentinogenesis imperfecta and dentin dysplasia revisited: problems in classification. J. Oral Pathol., Copenhagen, v. 17, no. 9-10, p. 547-553, Nov. 1988.

NOLLA, C.M. The development of permanent teeth. J. Dent. Child., Fulton, MO, v. 27, p. 254-266, 1960.

OLIVEIRA, B.H.; MILBOURNE, P. Fluorose dentária em incisivos superiores permanentes em crianças de escola pública do Rio de Janeiro, RJ. R. Saúde Pública, São Paulo, v. 35, no. 3, p. 276-282, jun. 2001.

PINHEIRO, I.V.A. et al. Lesões brancas no esmalte dentário: como diferenciá-las e tratá-las. R. Bras. Patol. Oral, Natal, v. 2, n. 1, p.11-18, jan./mar. 2003.

REGEZI, J.A.; SCIUBBA, J.J. Patologia bucal: correlações clinicopatológicas. 3.ed. Rio de Janeiro: Guanabara Koogan, 2000. p. 405-429.

SEOW, W.K.; AMARATUNGE, A. The effect of acid-etching on enamel from different clinical variants of amelogenesis imperfecta: an SEM study. Pediatr. Dent., Chicago, v. 20, no. 1, p. 37-42. Jan./Feb. 1998.

SEOW, W.K.; HUMPHRYS, C.; TUDEHOPE, D.I. Increased prevalence of developmental dental defects in low birth-weight, prematurely born children: a controlled study. Pediatr. Dent., Chicago, v. 9, no. 3, p. 221-225. Sept. 1987.

SHAFER, W.G.; HINE, M.K.; LEVY, B.M. Tratado de patologia bucal. 4. ed. Rio de Janeiro: Guanabara Koogan, 1987. p.1-79.

NEVILLE, B.W. et al. Patologia oral e maxilofacial. Rio de Janeiro: Guanabara Koogan, 1998. p.43-92. 